\title{
Interface Error Analysis for Numerical Wave Propagation
}

\author{
William W. Symes, Tetyana Vdovina* \\ * The Rice Inversion Project, Department of Computational and Applied Mathematics, \\ Rice University, Houston TX 77251-1892 USA, email symes@caam.rice.edu, \\ vdovina@caam.rice.edu.
}

(October 5, 2008)

Running head: Interface Error Analysis

\begin{abstract}
The numerical error associated with finite-difference simulation of wave propagation in discontinuous media consists of two components. The first component is a higher order error that leads to grid dispersion; it can be controlled by higher-order methods. The second component results from misalignment between numerical grids and material interfaces. We provide an explicit estimate of the interface misalignment error for the second order in time and space staggered finite-difference scheme applied to the acoustic wave equation. Our analysis, confirmed by numerical experiments, demonstrates that the interface error results in a first-order time shift proportional to the distance between the interface and computational grids. A two-dimensional experiment shows that the interface error cannot be suppressed by higher-order methods and indicates that our one-dimensional analysis gives a good prediction about the behavior of the numerical solution in higher dimensions.
\end{abstract}




\section{INTRODUCTION}

Finite-difference modeling of seismic wave propagation has long been used to enhance the understanding and interpretation of wave phenomena observed in seismology, acoustics, and electrodynamics. The properties of explicit finite-difference schemes on regular uniform meshes have been studied from various prospectives and the behavior of finite-difference solutions in homogeneous media is now well understood. Theoretical analysis shows that as time and space steps go to zero, finite-difference solutions converge at definite rate to the exact solutions, provided that the finite-difference scheme is stable and material parameters are smooth. For applications to seismology, however, this understanding does not suffice. The mechanical characteristics of actual rocks generally vary significantly on all spatial scales (see, for example, Bourbie et al. (1987), Dobrin and Savit (1988), Walden and Hosken (1986)). That is, they are essentially discontinuous as functions of spatial location. Thus the theory just described does not apply to faithful models of wave propagation in the Earth. Discontinuous models also appear in acoustic and electromagnetic simulation applications.

In this paper, we study a simple special case of discontinuously heterogeneous material models, in which homogeneous or smoothly heterogeneous material regions are separated by smooth or piecewise smooth interfaces across which the coefficients in the wave equation may be discontinuous. Even in this simpler setting, it is known that a difference scheme of formal $p$-th order accuracy typically exhibits only first-order converngence. Brown (1984) shows that the computational error associated with an interface problems consists of two components. In addition to the higher order component that corresponds to the truncation error of the homogeneous problem, there is a first-order error that is due to the material interface.

Gustafsson and Wahlund (2004) study the interaction between the material discontinuity error and higher order error for the time compact staggered finite-difference schemes of the 2nd and 4th order in time and space. They show that as in the homogeneous case, grid dispersion is due to a higher order component of the error which grows with time and/or frequency for any fixed number of grid points per wavelength. The first-order interface error, on the other hand, is independent of time and/or frequency. This means that if the simulation is run for long time periods, the grid dispersion error becomes dominant. Gustafsson and Wahlund (2004) focus on demonstrating that the grid dispersion error can 
be controlled by the use of higher-order operators. However, the application of higherorder operators does not eliminate the interface error which becomes dominant once the higher-order error is controlled.

The goal of this work is to measure the interface error in typical conditions of exploration seismic modeling. We show that if an input model is described only as a grid of values (i.e., the locations of the interfaces are specified only up to grid cell resolution), accurate interaction of the numerical wave with an interface cannot be guaranteed. The exact location of the interface determines the arrival time of the wave. Since the interface can lie anywhere in a grid cell and the coefficients of a finite-difference scheme are insensitive to its exact position, the numerical wave can be shifted from the actual wave by as much as the travel time through a cell. When several interfaces are present, the error can be cumulative.

We quantitatively evaluate the first-order interface misalignment error for the second order in time and space staggered finite-difference scheme applied to the pressure-velocity formulation of the acoustic wave equation (see Virieux (1984), Virieux (1986)). In our analysis, we follow the pattern of Gustafsson and Wahlund (2004) and break the error into first-order and higher order components. However, while Gustafsson and Wahlund (2004) focus on supressing the higher-order error, we provide an explicit estimate for the first-order interface misalignment error and show that it may be large enough to dominate other errors. Staggered finite difference methods are characterized by the presence of two computational grids (in the one-dimensional case). Assuming that the interface is located between pressure and velocity grid nodes, we show that, for any fixed frequency, the pressure and velocity errors are proportional to the distance between the interface and the nearest pressure and velocity grid points, respectively. It follows, that even if the interface is aligned with one of the computational grids, the misalignment with the second grid results in a non-zero error proportional to the time step. To first order, this error manifests itself as a non-zero time shift, for which we give an explicit expression.

In the following section, we introduce the model problem, describe the second-order in time and space staggered finite-difference scheme, and perform the analysis of the interface error. Then we present two numerical examples followed by a discussion that illustrate our results in typical conditions of exploration seismic modeling. The numerical examples are consistent with formal mathematical analysis and demonstrate that the interface error 
amounts to a time shift in traces.

\section{INTERFACE ERROR ANALYSIS}

We consider the following formulation of the acoustic wave equation in terms of pressure and velocity:

$$
\begin{aligned}
\frac{1}{\kappa(x)} \frac{\partial p(x, t)}{\partial t} & =-\frac{\partial v(x, t)}{\partial x} \\
\rho(x) \frac{\partial v(x, t)}{\partial t} & =-\frac{\partial p(x, t)}{\partial x}
\end{aligned}
$$

in a two-layer medium

$$
(\kappa(x), \rho(x))= \begin{cases}\left(\kappa_{L}, \rho_{L}\right) & \text { for } x \leq 0 \\ \left(\kappa_{R}, \rho_{R}\right) & \text { for } x>0\end{cases}
$$

where $\kappa$ and $\rho$ denote the bulk modulus and density, respectively. Speed of the wave is $c_{L}=\sqrt{\kappa_{L} / \rho_{L}}$ to the left from the interface and $c_{R}=\sqrt{\kappa_{R} / \rho_{R}}$ to the right from the interface. We assume that an incident wave with a unit amplitude propagates in the medium to the left from the interface. After an interaction with the interface, the wave will be decomposed into a transmitted and reflected waves with amplitudes $T$ and $R$. The solution of problem (1)-(2) is a linear combination of the incident, transmitted, and reflected waves:

$$
\begin{aligned}
& p(x, t)= \begin{cases}c_{L} \rho_{L} e^{i \omega\left(t-x / c_{L}\right)}-c_{L} \rho_{L} R e^{i \omega\left(t+x / c_{L}\right)} & \text { for } x \leq 0, \\
c_{R} \rho_{R} T e^{i \omega\left(t-x / c_{R}\right)} & \text { for } x>0,\end{cases} \\
& v(x, t)= \begin{cases}e^{i \omega\left(t-x / c_{L}\right)}+R e^{i \omega\left(t+x / c_{L}\right)} & \text { for } x \leq 0, \\
T e^{i \omega\left(t-x / c_{R}\right)} & \text { for } x>0 .\end{cases}
\end{aligned}
$$

Continuity of the pressure and velocity solutions at the interface yields the following system of equations for reflection and transmission coefficients $R$ and $T$ :

$$
\left[\begin{array}{cc}
c_{L} \rho_{L} & c_{R} \rho_{R} \\
-1 & 1
\end{array}\right]\left[\begin{array}{c}
R \\
T
\end{array}\right]=\left[\begin{array}{c}
c_{L} \rho_{L} \\
1
\end{array}\right]
$$


Denoting the coefficient matrix by $A$, the vector of unknowns by $\sigma$, and the right-hand side vector by $g$, we rewrite system (6) as follows:

$$
A \sigma=g
$$

Next, we describe the continuous in time second-order in space finite-difference approximation of problem (1)-(2) and construct the numerical solution. The setup yields a staggered grid (decimated leapfrog) method when the continuous time evolution is replaced by a centered difference method (Virieux (1986)). Our analysis neglects time discretization error. Following Gustafsson and Wahlund (2004), we introduce parameter $\delta, 0 \leq \delta \leq 1 / 2$ that measures the shift of the grid relative to the interface (see Figure 1).

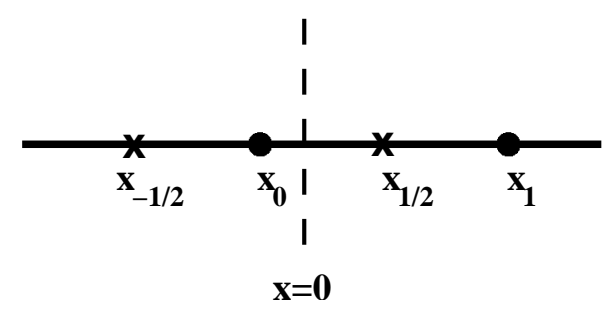

Figure 1: Location of the interface. Circles represent pressure (main) grid nodes. Crosses represent velocity (staggered) grid nodes.

The grid nodes and values are then defined as follows:

$$
\begin{aligned}
& x_{j}=(j-\delta) h \text { for } j=0, \pm 1 / 2, \pm 1, \ldots, \\
& p_{j}=p\left(x_{j}, t\right) \\
& v_{j+1 / 2}=v\left(x_{j+1 / 2}, t\right) .
\end{aligned}
$$

The second-order semi-discrete scheme is

$$
\begin{aligned}
\frac{\partial p_{j}}{\partial t} & =-\frac{\kappa_{j}}{h}\left(v_{j+1 / 2}-v_{j-1 / 2}\right), \\
\frac{\partial v_{j+1 / 2}}{\partial t} & =-\frac{1}{\rho_{j+1 / 2} h}\left(p_{j+1}-p_{j}\right)
\end{aligned}
$$


and the corresponding solutions are:

$$
\begin{gathered}
p_{j}= \begin{cases}c_{L} \rho_{L} e^{i\left(\omega t-(j-\delta) h k_{L}\right)}-c_{L} \rho_{L} R_{h} e^{i\left(\omega t+(j-\delta) h k_{L}\right)} & \text { for } x \leq 0, \\
c_{R} \rho_{R} T_{h} e^{i\left(\omega t-(j-\delta) h k_{R}\right)} & \text { for } x>0,\end{cases} \\
v_{j+1 / 2}= \begin{cases}e^{i\left(\omega t-(j+1 / 2-\delta) h k_{L}\right)}+R_{h} e^{i\left(\omega t+(j+1 / 2-\delta) h k_{L}\right)} & \text { for } x \leq 0, \\
T_{h} e^{i\left(\omega t-(j+1 / 2-\delta) h k_{R}\right)} & \text { for } x>0,\end{cases}
\end{gathered}
$$

where $k_{L}$ and $k_{R}$ are the wave numbers for the medium to the left and to the right of the interface. As before, we use the continuity condition to determine the system for numerical reflection and transmission coefficients:

$$
\begin{aligned}
c_{L} \rho_{L} R_{h} e^{-i \delta h k_{L}}+c_{R} \rho_{R} T_{h} e^{i \delta h k_{R}} & =c_{L} \rho_{L} e^{i \delta h k_{L}}, \\
-R_{h} e^{i(1 / 2-\delta) h k_{L}}+T_{h} e^{-i(1 / 2-\delta) h k_{R}} & =e^{-i(1 / 2-\delta) h k_{L}} .
\end{aligned}
$$

Expanding exponential functions to the first order, we rewrite (15)-(16) in the matrix form:

$$
\left[\begin{array}{cc}
c_{L} \rho_{L}\left(1-i \delta h k_{L}\right) & c_{R} \rho_{R}\left(1+i \delta h k_{R}\right) \\
-1-i(1 / 2-\delta) h k_{L} & 1-i(1 / 2-\delta) h k_{R}
\end{array}\right]\left[\begin{array}{c}
R_{h} \\
T_{h}
\end{array}\right]=\left[\begin{array}{c}
c_{L} \rho_{L}\left(1+i \delta h k_{L}\right) \\
1-i(1 / 2-\delta) h k_{L}
\end{array}\right]+O\left(h^{2}\right) .(17
$$

Wave numbers $k_{L}$ and $k_{R}$ can be obtained from a standard plane wave analysis (see, for example, Cohen (2002)):

$$
\begin{aligned}
& k_{L}=\frac{2}{h} \arcsin \frac{\omega h}{2 c_{L}}=\frac{\omega}{c_{L}}+O\left(h^{2}\right), \\
& k_{R}=\frac{2}{h} \arcsin \frac{\omega h}{2 c_{R}}=\frac{\omega}{c_{R}}+O\left(h^{2}\right) .
\end{aligned}
$$

Using the last equations in (17) and introducing vectors

$$
\sigma_{h}=\left[\begin{array}{c}
R_{h} \\
T_{h}
\end{array}\right], \quad g_{h}=i h \omega\left[\begin{array}{c}
\rho_{L} \delta \\
(\delta-1 / 2) / c_{L}
\end{array}\right]
$$

and matrix

$$
A_{h}=i h \omega\left[\begin{array}{cc}
-\rho_{L} \delta & \rho_{R} \delta \\
(\delta-1 / 2) / c_{L} & (\delta-1 / 2) / c_{R}
\end{array}\right]
$$


we write system (17) in the following form:

$$
\left(A+A_{h}\right) \sigma_{h}=g+g_{h}+O\left(h^{2}\right) .
$$

Numerical solution $\sigma_{h}$ is a sum of the exact solution $\sigma$ and truncation error $\tau$. Therefore, equation (22) becomes:

$$
A \sigma+\left(A+A_{h}\right) \tau=g-A_{h} \sigma+g_{h}+O\left(h^{2}\right) .
$$

Since $\sigma$ satisfies $A \sigma=g$, truncation error $\tau$ satisfies the remaining equation:

$$
\left(A+A_{h}\right) \tau=g_{h}-A_{h} \sigma+O\left(h^{2}\right) .
$$

The determinant of $A+A_{h}$ is:

$$
D=c_{L} \rho_{L}+c_{R} \rho_{R}+O(h \omega \delta) .
$$

Therefore, there is a solution of equation (24):

$$
\tau=\left(A+A_{h}\right)^{-1}\left(g_{h}-A_{h} \sigma\right)+O\left(h^{2}\right),
$$

where

$$
g_{h}-A_{h} \sigma=i h \omega\left[\begin{array}{c}
\delta \rho_{L}(1+R)-\delta \rho_{R} T \\
\frac{\delta-1 / 2}{c_{L}}(1-R)-\frac{\delta-1 / 2}{c_{R}} T
\end{array}\right] .
$$

Using the fact that $1-R=\frac{2 c_{R} \rho_{R}}{c_{L} \rho_{L}+c_{R} \rho_{R}}$ and $T=1+R=\frac{2 c_{L} \rho_{L}}{c_{L} \rho_{L}+c_{R} \rho_{R}}$, we rewrite vector (27) as follows:

$$
g_{h}-A_{h} \sigma=\frac{2 i h \omega}{c_{L} \rho_{L}+c_{R} \rho_{R}}\left[\begin{array}{c}
\delta c_{L} \rho_{L}\left(\rho_{L}-\rho_{R}\right) \\
(\delta-1 / 2) \frac{c_{R}^{2} \rho_{R}-c_{L}^{2} \rho_{L}}{c_{L} c_{R}}
\end{array}\right]
$$

Then, equation (26) becomes:

$$
\begin{aligned}
\tau & =\frac{2 i h \omega}{\left(c_{L} \rho_{L}+c_{R} \rho_{R}\right)^{2}}\left[\begin{array}{cc}
1+i(\delta-1 / 2) \frac{h \omega}{c_{R}} & -c_{R} \rho_{R}\left(1+\frac{i \delta h \omega}{c_{R}}\right) \\
1-i(\delta-1 / 2) \frac{h \omega}{c_{L}} & c_{L} \rho_{L}\left(1-\frac{i \delta h \omega}{c_{L}}\right)
\end{array}\right]\left[\begin{array}{c}
\delta c_{L} \rho_{L}\left(\rho_{L}-\rho_{R}\right) \\
(\delta-1 / 2) \frac{c_{R}^{2} \rho_{R}-c_{L}^{2} \rho_{L}}{c_{L} c_{R}}
\end{array}\right]+O\left(h^{2}\right) \\
& =\frac{2 i h \omega}{\left(c_{L} \rho_{L}+c_{R} \rho_{R}\right)^{2}}\left[\begin{array}{cc}
1 & -c_{R} \rho_{R} \\
1 & c_{L} \rho_{L}
\end{array}\right]\left[\begin{array}{c}
\delta c_{L} \rho_{L}\left(\rho_{L}-\rho_{R}\right) \\
(\delta-1 / 2) \frac{c_{R}^{2} \rho_{R}-c_{L}^{2} \rho_{L}}{c_{L} c_{R}}
\end{array}\right]+O\left(h^{2} \omega^{2} \delta^{2}\right),
\end{aligned}
$$


where we have dropped the higher-order terms. Multiplying, we obtain:

$$
\begin{aligned}
\tau & =\frac{2 i h \omega}{\left(c_{L} \rho_{L}+c_{R} \rho_{R}\right)^{2}}\left[\begin{array}{c}
\delta c_{L} \rho_{L}\left(\rho_{L}-\rho_{R}\right)-(\delta-1 / 2) \frac{\rho_{R}}{c_{L}}\left(c_{R}^{2} \rho_{R}-c_{L}^{2} \rho_{L}\right) \\
\delta c_{L} \rho_{L}\left(\rho_{L}-\rho_{R}\right)+(\delta-1 / 2) \frac{\rho_{L}}{c_{R}}\left(c_{R}^{2} \rho_{R}-c_{L}^{2} \rho_{L}\right)
\end{array}\right] \\
& +O\left(h^{2} \omega^{2} \delta^{2}\right) .
\end{aligned}
$$

Using the fact that $e^{a}=1+a+O\left(a^{2}\right)$, we obtain for the numerical reflection coefficient:

$$
\begin{aligned}
R_{h} & =R\left(1+\frac{2 i h \omega}{R\left(c_{L} \rho_{L}+c_{R} \rho_{R}\right)^{2}}\left(\delta c_{L} \rho_{L}\left(\rho_{L}-\rho_{R}\right)-(\delta-1 / 2) \frac{\rho_{R}}{c_{L}}\left(c_{R}^{2} \rho_{R}-c_{L}^{2} \rho_{L}\right)\right)\right)+O\left(h^{2} \omega^{2} \delta^{2}\right) \\
& =R \exp \left\{\frac{2 i h \omega\left(\delta c_{L} \rho_{L}\left(\rho_{L}-\rho_{R}\right)-(\delta-1 / 2) \frac{\rho_{R}}{c_{L}}\left(c_{R}^{2} \rho_{R}-c_{L}^{2} \rho_{L}\right)\right)}{c_{L}^{2} \rho_{L}^{2}-c_{R}^{2} \rho_{R}^{2}}\right\}+O\left(h^{2} \omega^{2} \delta^{2}\right) .
\end{aligned}
$$

Similarly, for the numerical transmission coefficient we have:

$$
T_{h}=T \exp \left\{\frac{2 i h \omega\left(\delta c_{L} \rho_{L}\left(\rho_{L}-\rho_{R}\right)-(\delta-1 / 2) \frac{\rho_{R}}{c_{L}}\left(c_{R}^{2} \rho_{R}-c_{L}^{2} \rho_{L}\right)\right)}{2 c_{L} \rho_{L}\left(c_{L} \rho_{L}+c_{R} \rho_{R}\right)}\right\}+O\left(h^{2} \omega^{2} \delta^{2}\right) .
$$

Equations (30) and (31) imply that the numerical reflection and transmission coefficients have the same amplitude as the exact coefficients and differ from $R$ and $T$ by a phase shift up to a second-order error. After centered difference time discretization, the space step $h$ is related to the time step by $\lambda h=\Delta t$, which is the standard stability condition with $\lambda$ confined to the interval $0<\lambda<1 / c$. Therefore, the phase shift is proportional to the time step and is non-zero and of the first order in $h \omega$ even if the interface is aligned with one of the computational grids.

\section{NUMERICAL EXPERIMENTS}

In this section, we present two numerical experiments which support our contention that the first order error is endemic to staggered-grid finite-difference simulation with discontinuous coefficients and may easily dominate other error components. The first experiment illustrates the error analysis performed in the previous section. Using a one-dimensional model with a single interface, we compare the second-order in time and space staggered finite-difference solution to the analytical solution. We change the position of the interface relative to both main and staggered grids and show that while the analytical solution captures the interface location exactly, the first-order error in the numerical solution results 
in a time-shift even when the interface is aligned with a computational grid. The second experiment suggests that this phenomenon is not confined to $1 \mathrm{D}$ even though our analysis is. Further, we use this example to show that higher-order methods do not reduce the first-order error caused by coefficient discontinuity.

In the first experiment, we solve problem (1)-(2) in the domain $0 \leq t \leq 600 \mathrm{~ms}$, $0 \leq x \leq 1800 \mathrm{~m}$ with zero boundary conditions. We choose the other inputs to the simulation to ensure that propagation in a homogeneous medium over the space-time region would be quite accurate. Thus the simulation error should consist mostly of the interface error analyzed in the last section.

We assume that the interface is located at $x=x_{b}$ and set $\rho_{L}=2100 \mathrm{~kg} / \mathrm{m}^{3}, c_{L}=$ $2.3 \mathrm{~m} / \mathrm{ms}$ and $\rho_{R}=2300 \mathrm{~kg} / \mathrm{m}^{3}, c_{L}=3.0 \mathrm{~m} / \mathrm{ms}$. The analytical solution is:

$$
\begin{aligned}
& p(t, x)= \begin{cases}f\left(t-x / c_{L}\right)-\frac{c_{L} \rho_{L}-c_{R} \rho_{R}}{c_{L} \rho_{L}+c_{R} \rho_{R}} f\left(t+x / c_{L}\right) & \text { for } x \leq x_{b}, \\
\frac{2 c_{R} \rho_{R}}{c_{L} \rho_{L}+c_{R} \rho_{R}} f\left(t-x / c_{R}\right) & \text { for } x>x_{b},\end{cases} \\
& v(t, x)= \begin{cases}\left(f\left(t-x / c_{L}\right)+\frac{c_{L} \rho_{L}-c_{R} \rho_{R}}{c_{L} \rho_{L}+c_{R} \rho_{R}} f\left(t+x / c_{L}\right)\right) / c_{L} \rho_{L} & \text { for } x \leq x_{b}, \\
\frac{2}{c_{L} \rho_{L}+c_{R} \rho_{R}} f\left(t-x / c_{R}\right) & \text { for } x>x_{b},\end{cases}
\end{aligned}
$$

where $f$ is a Ricker's wavelet with central frequency $f_{0}=10 \mathrm{~Hz}$ :

$$
f(t)=\left(1-2\left(\pi f_{0}\left(t-t_{0}\right)\right)^{2}\right) e^{-\left(\pi f_{0}\left(t-t_{0}\right)\right)^{2}} .
$$

We discretize the domain into 360 grid points and set interface equal to $x_{b}=900.0,901.25$, 902.5, 903.75, and $905.0 \mathrm{~m}$. Interfaces $x_{b}=900.0 \mathrm{~m}$ and $x_{b}=905.0 \mathrm{~m}$ coincide with pressure (main) grid nodes, interface $x_{b}=902.5$ goes through the velocity (staggered) grid node, and interfaces $x_{b}=901.25 \mathrm{~m}$ and $x_{b}=903.75 \mathrm{~m}$ are located at $1.25 \mathrm{~m}$ from both the pressure and velocity grid nodes (see Figure 2 for details).

Figures 3 and 4 show traces of the analytical and numerical pressure and velocity solutions at $500 \mathrm{~m}$. Figures 5 and 6 show the same traces between 405 and $440 \mathrm{~ms}$. Blue, cyan, green, brown, and magenta curves describe the analytical solutions that correspond to each of the five interface locations described above. As expected, the analytical solutions are at the same distance from each other and capture the locations of the interfaces exactly. The black curve represents numerical solutions that correspond to interface locations $x_{b}=900.0$ 


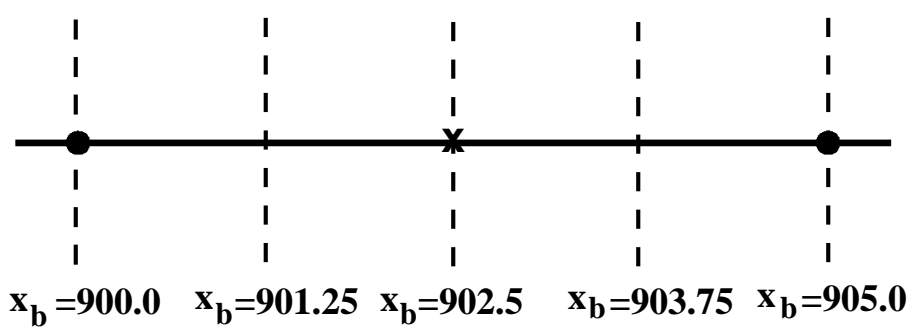

Figure 2: Locations of the interfaces. Circles represent pressure (main) grid nodes. Crosses represent velocity (staggered) grid nodes.

and $901.25 \mathrm{~m}$. Similarly, red curve describes coinciding numerical solutions that correspond to interface locations 902.5 and $903.75 \mathrm{~m}$. Finally, orange curve corresponds to the numerical solution with interface located at $x_{b}=905.0 \mathrm{~m}$. It is clear from Figures 5 and 6 that all numerical solutions are shifted from the respective analytical solutions. The biggest time shift of about $1 \mathrm{~ms}$ occurs between solutions that correspond to interfaces located at $x_{b}=900.0 \mathrm{~m}$ and $x_{b}=905.0 \mathrm{~m}$ (compare black and blue curves or orange and magenta curves). Figure 7 shows plots of the analytic and numerical solutions and difference between them for the interface location $x_{b}=900.0 \mathrm{~m}$ and time interval $300 \mathrm{~ms} \leq t \leq 600 \mathrm{~ms}$ (i.e., the reflected wave only). RMS error for the reflected wave is $16 \%$. The time shift is much smaller for the interface locations $x_{b}=902.5$ and $x_{b}=903.75 \mathrm{~m}$ (compare red and green curves or red and brown curves). RMS error in this case is approximately $4 \%$.

In the second experiment, we present results derived from a simple model of a dome structure in 2D. Figure 8 displays the velocity (top) and density (bottom) fields for this model. The wave propagation is forced by a point dilatational source located at point $\mathbf{x}_{s}$. The force is described by a right-hand side in the Newton's law (equation (2)):

$$
f(t, \mathbf{x})=g(t) \nabla \delta\left(\mathbf{x}-\mathbf{x}_{s}\right)
$$

An alternative representation of the energy source as a right-hand side in the constitutive law (equation (1)) is also possible and produces the same field away from the source point. We choose $g(t)$ to be the Gaussian wavelet scaled as follows:

$$
g(t)=\frac{2 c^{2} r_{0}}{\pi f_{0}^{2}} e^{-\pi f_{0} t}
$$




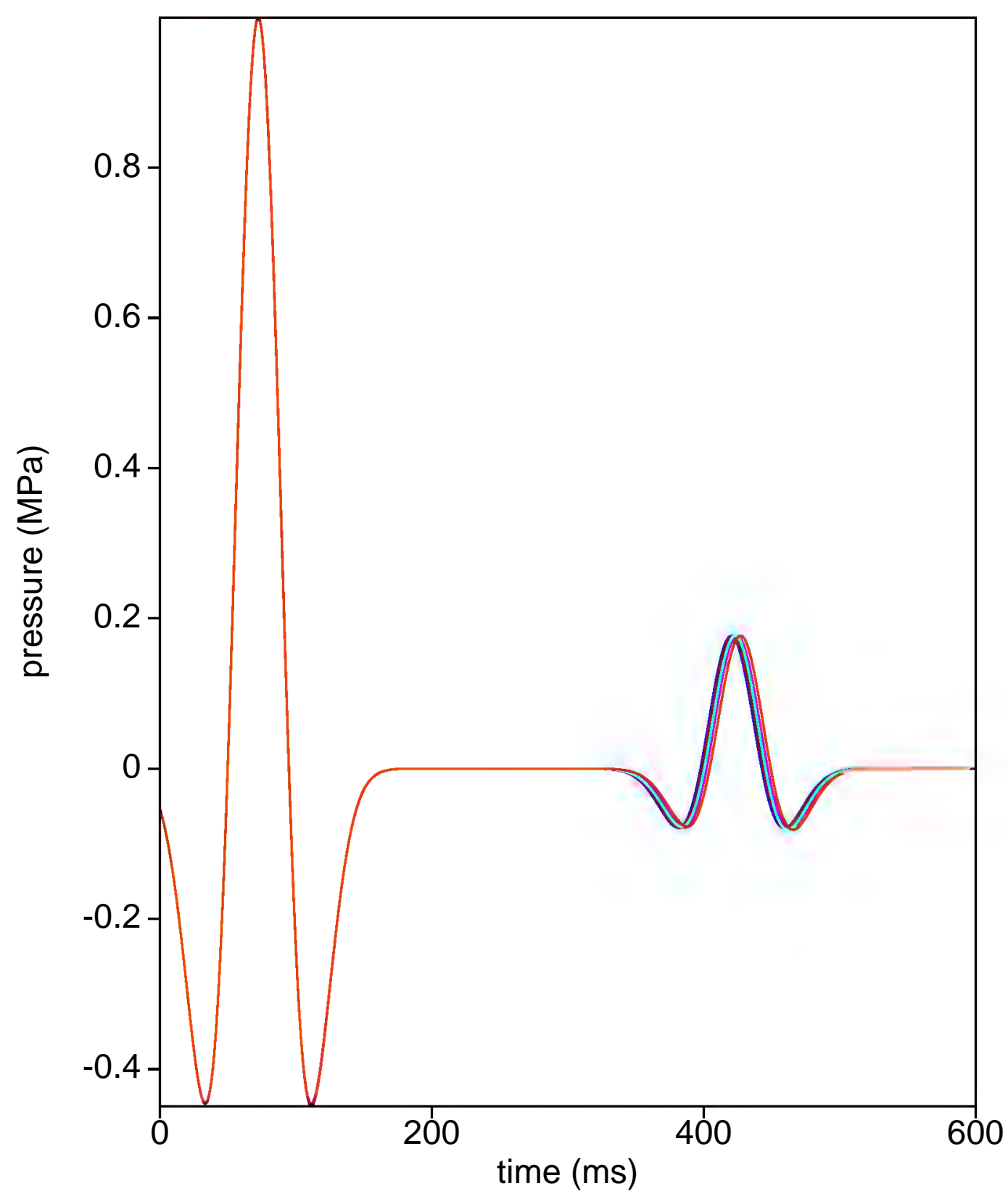

Figure 3: Traces of the analytical and numerical pressure solutions at $500 \mathrm{~m}$. Analytical solutions: blue for $x_{b}=900.0 \mathrm{~m}$, cyan for $x_{b}=901.25 \mathrm{~m}$, green for $x_{b}=902.5 \mathrm{~m}$, brown for $x_{b}=903.75 \mathrm{~m}$, magenta for $x_{b}=905.0 \mathrm{~m}$. Numerical solutions: black for $x_{b}=900.0 \mathrm{~m}$ 11 and $x_{b}=901.25 \mathrm{~m}$, red for $x_{b}=902.5 \mathrm{~m}$ and $x_{b}=903.75 \mathrm{~m}$, orange for $x_{b}=905.0 \mathrm{~m}$. 


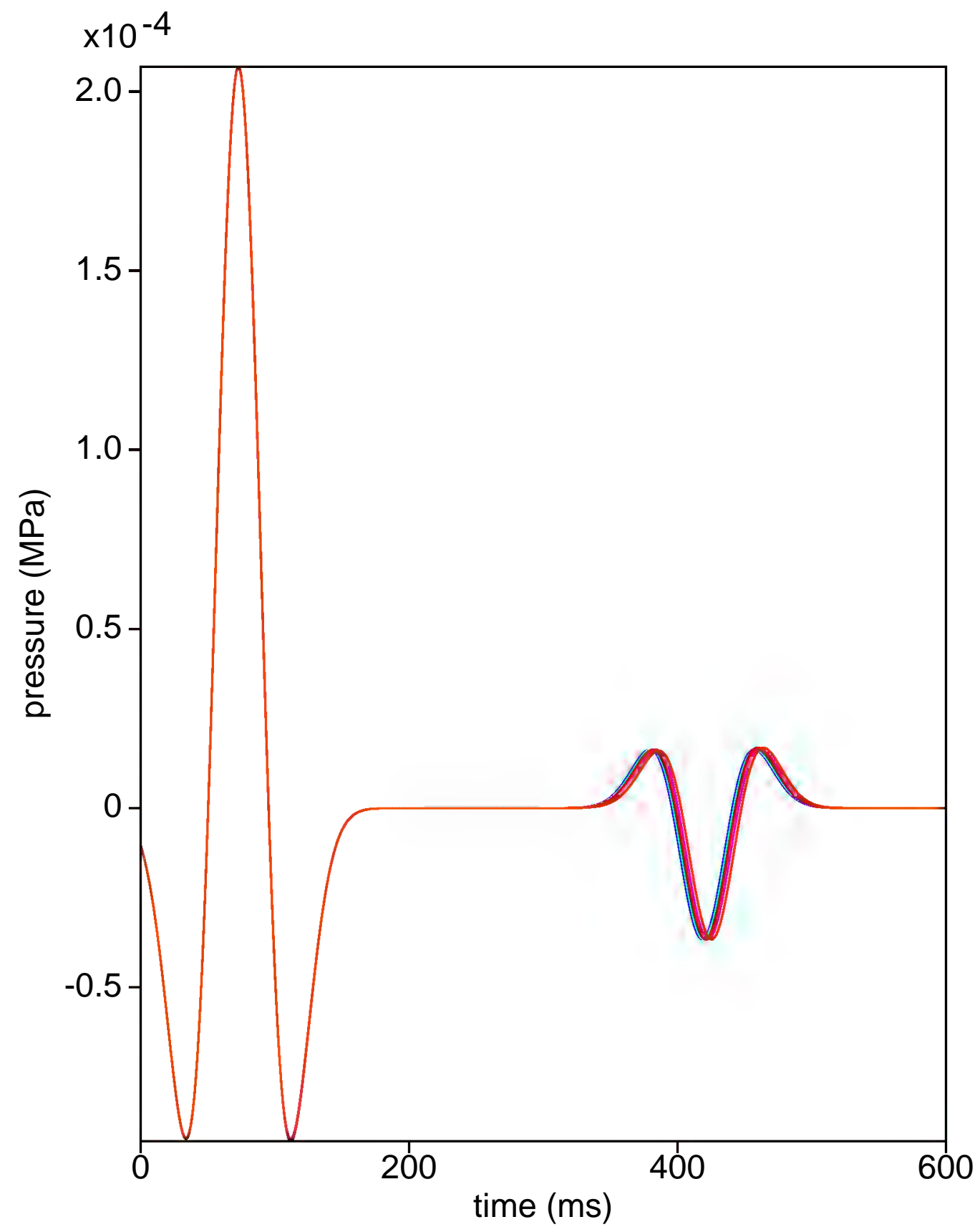

Figure 4: Traces of the analytical and numerical velocity solutions at $500 \mathrm{~m}$. Analytical solutions: blue for $x_{b}=900.0 \mathrm{~m}$, cyan for $x_{b}=901.25 \mathrm{~m}$, green for $x_{b}=902.5 \mathrm{~m}$, brown for $x_{b}=903.75 \mathrm{~m}$, magenta for $x_{b}=905.0 \mathrm{~m}$. Numerical solutions: black for $x_{b}=900.0 \mathrm{~m}$ 12 and $x_{b}=901.25 \mathrm{~m}$, red for $x_{b}=902.5 \mathrm{~m}$ and $x_{b}=903.75 \mathrm{~m}$, orange for $x_{b}=905.0 \mathrm{~m}$. 


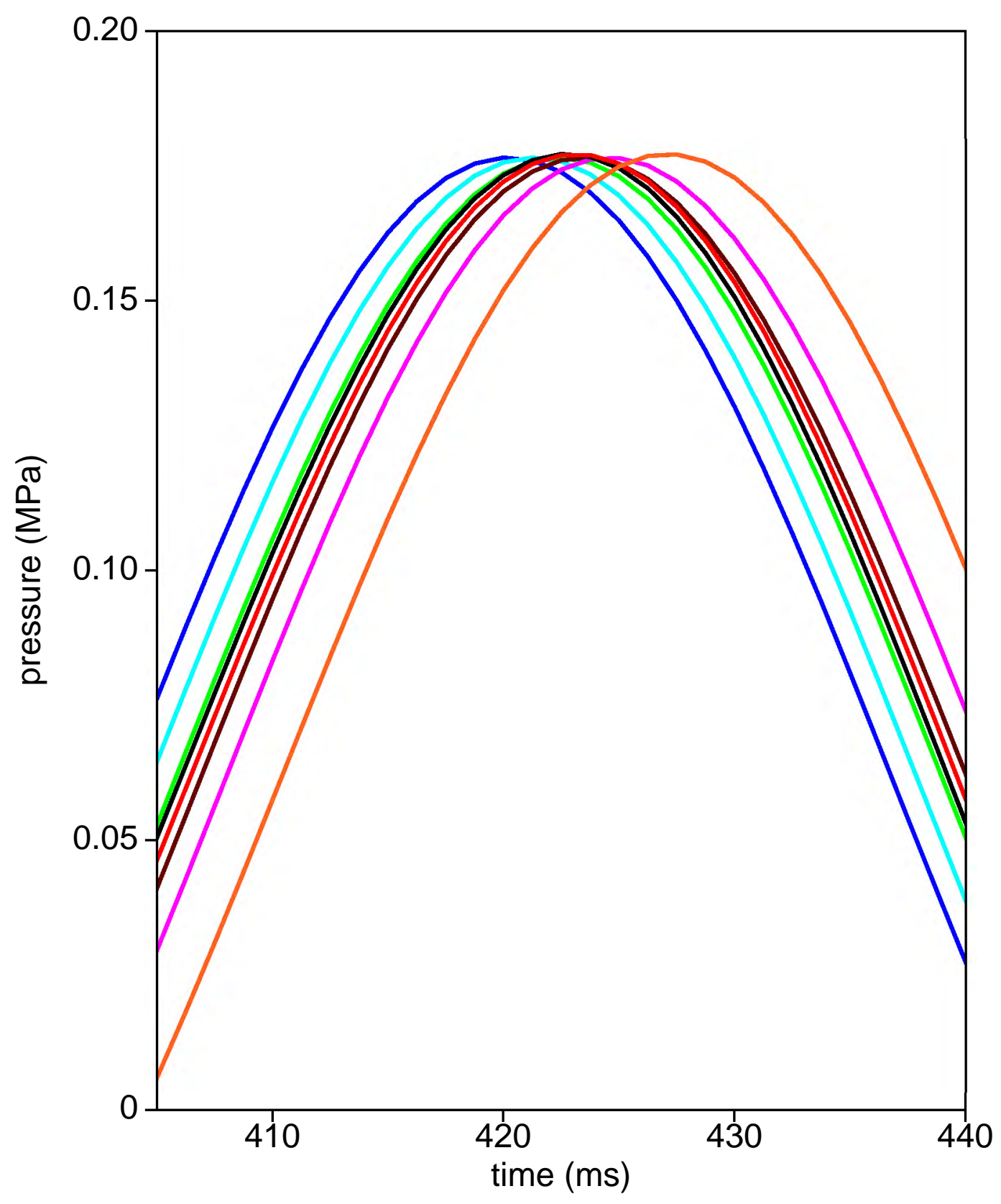

Figure 5: Traces of the analytical and numerical solutions at $500 \mathrm{~m}$. Analytical solutions: blue for $x_{b}=900.0 \mathrm{~m}$, cyan for $x_{b}=901.25 \mathrm{~m}$, green for $x_{b}=902.5 \mathrm{~m}$, brown for $x_{b}=$ $903.75 \mathrm{~m}$, magenta for $x_{b}=905.0 \mathrm{~m}$. Numerical solutions: black for $x_{b}=900.0 \mathrm{~m}$ and 13 $x_{b}=901.25 \mathrm{~m}$, red for $x_{b}=902.5 \mathrm{~m}$ and $x_{b}=903.75 \mathrm{~m}$, orange for $x_{b}=905.0 \mathrm{~m}$. 


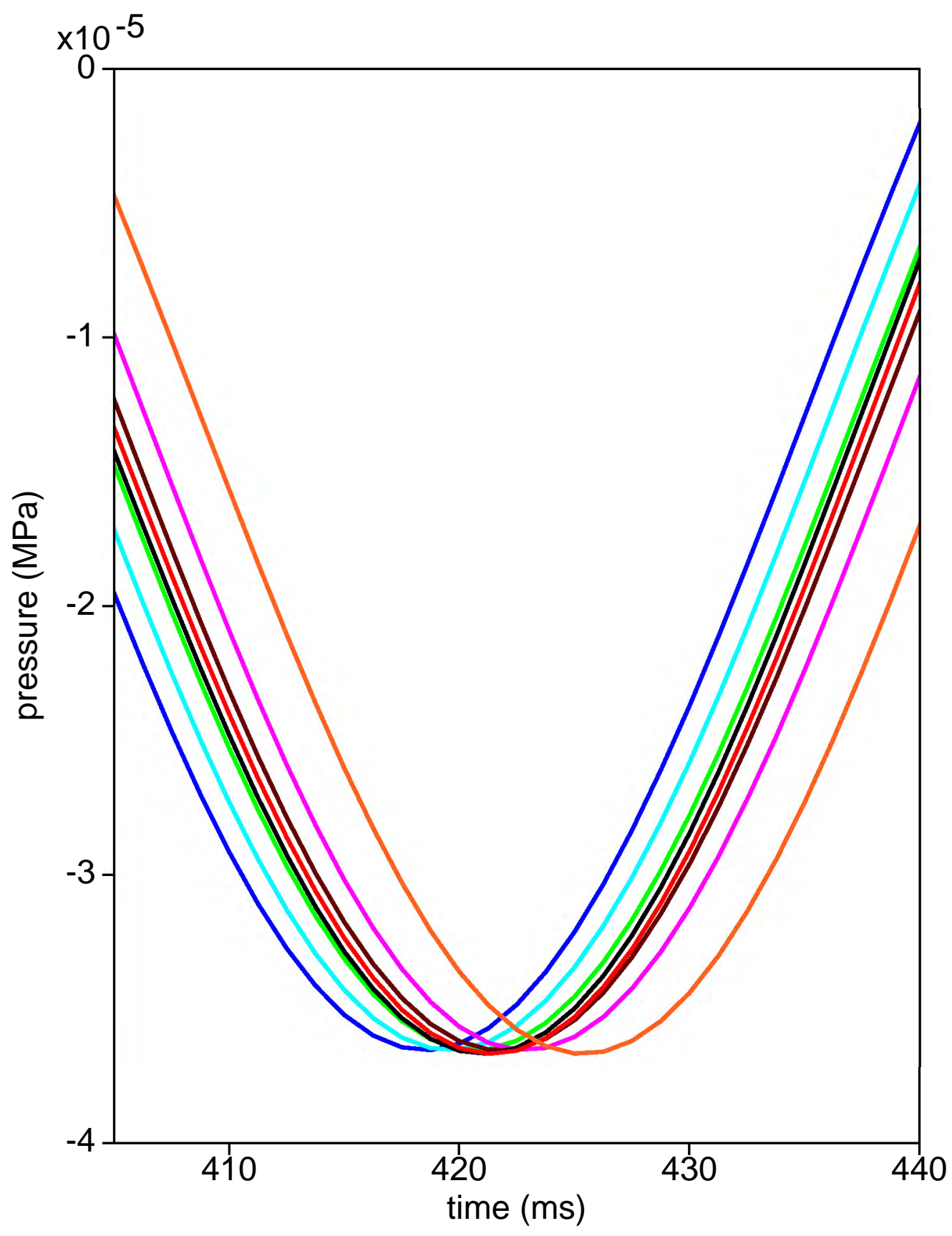

Figure 6: Traces of the analytical and numerical velocity solutions at $500 \mathrm{~m}$. Analytical solutions: blue for $x_{b}=900.0 \mathrm{~m}$, cyan for $x_{b}=901.25 \mathrm{~m}$, green for $x_{b}=902.5 \mathrm{~m}$, brown for $x_{b}=903.75 \mathrm{~m}$, magenta for $x_{b}=905.0 \mathrm{~m}$. Numerical solutions: black for $x_{b}=900.0 \mathrm{~m}$ 14 and $x_{b}=901.25 \mathrm{~m}$, red for $x_{b}=902.5 \mathrm{~m}$ and $x_{b}=903.75 \mathrm{~m}$, orange for $x_{b}=905.0 \mathrm{~m}$. 


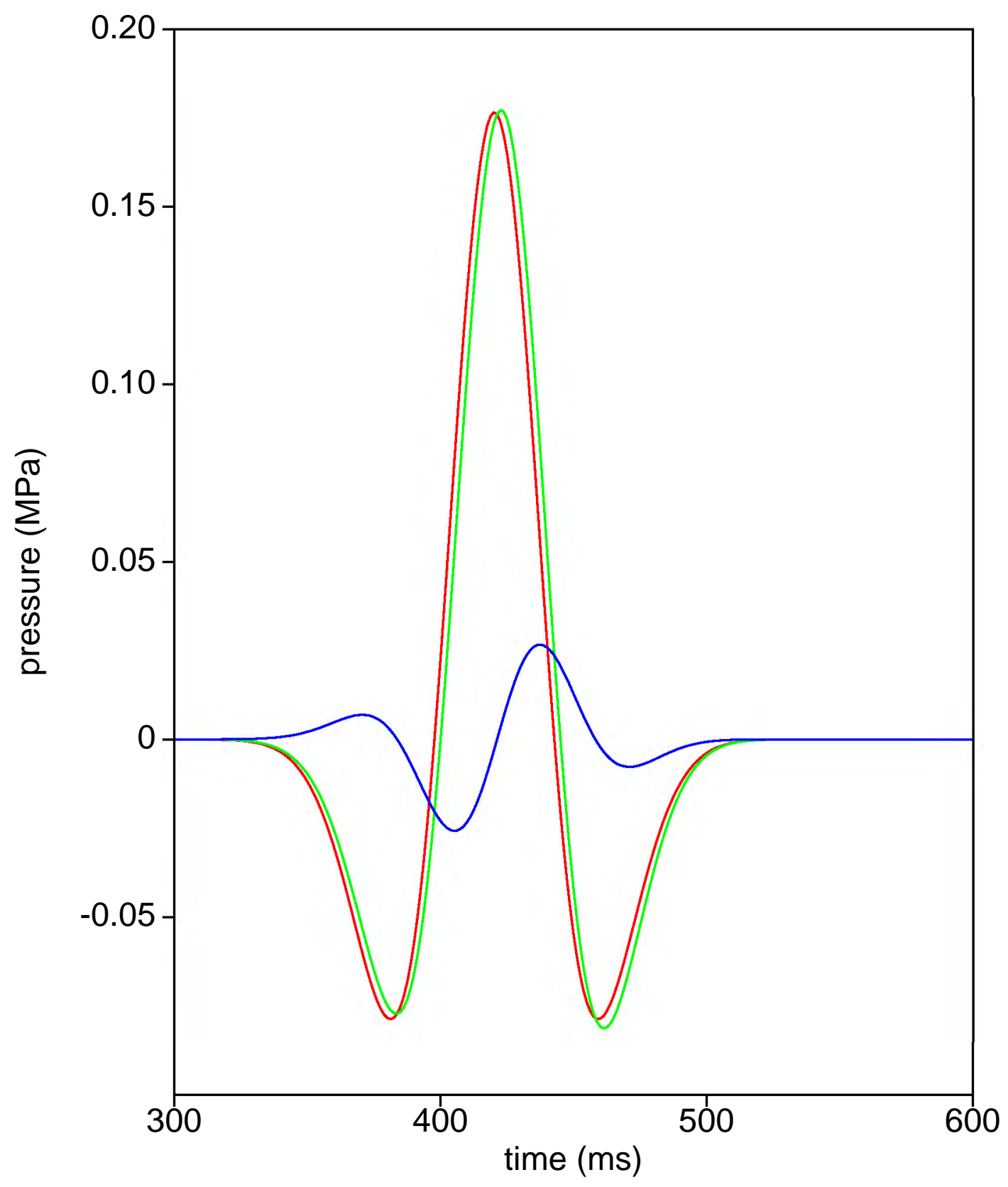

Figure 7: Comparison of traces for the interface located at $x_{b}=900 \mathrm{~m}$. Red: analytical solution. Green: numerical soltuion. Blue: difference between the analytical and numerical solutions. 
The Gaussian pulse (36) produces a propagating Ricker wavelet (second derivative of Gaussian) with peak frequency $f_{0}$ and a unit amplitude at distance $r_{0}$ from the source, if the simulations were conducted in $3 \mathrm{D}$. In $2 \mathrm{D}$, the pulse produced by equation (36) is more complex (a fractional derivative of the Gaussian).

We choose $f_{0}=15 \mathrm{~Hz}$, so that the wavelet has significant energy at $30 \mathrm{~Hz}$ or a wavelength of $50 \mathrm{~m}$ in the "water" layer at the top of the model. Source depth is $40 \mathrm{~m}$, receiver depth is $20 \mathrm{~m}$. The source position for this epxeriment is in the center, and the receiver configuration is symmetric split-spread, i.e. receivers are arranged symmetrically on a horizontal segment centered at the source.

Figures 9 and 10 show the results of a 4 th and 10th (spatial) order simulations on a $10 \mathrm{~m}$ grid. Some grid dispersion is evident in Figure 9, notably in the direct wave in the water, which is unsurprising as 5 grid points per wavelength is marginal for the 4th order scheme. As expected, a 10th order simulation shows little evidence of dispersion, which suggests that 5 grid points per wavelength is an adequate sample rate for this scheme. However, Figure 11 demonstrates that although the application of the 10th order scheme results in a dispersion-free solution, the interface error is still clearly present and manifests itself as a time-shift.

Figure 11 shows trace 100, at roughly $1 \mathrm{~km}$ offset, between 1100 and $1400 \mathrm{~ms}$. This event is the reflection from the dome, the nominal reflection point being near the top of the dome, in a gentle slope. The green curve is the $10 \mathrm{~m}$ grid 4 th order result, the blue curve is the $10 \mathrm{~m}$ grid 10 th order result. Even for this short travel time, the suppression of dispersive error from the former to the latter is evident. However, the black curve is the result of a $2.5 \mathrm{~m}$ grid 4 th order simulation, which is presumable closer to the truth. The difference between the $2.5 \mathrm{~m}$ result and either of the $10 \mathrm{~m}$ results is much greater then the difference between 4 th and 10th order at $10 \mathrm{~m}$. Further, the error consists of a time shift of approximately $10 \mathrm{~ms}$, which is roughly $15 \%$ of a wavelength at peak frequency.

The time shift is evidence of the interface error. The cell width of $10 \mathrm{~m}$ corresponds to $5 \mathrm{~ms}$ traveletime at the median velocity of $2 \mathrm{~km} / \mathrm{s}$ near the reflecting point. Since the reflection transits some part of the cell twice, up to $10 \mathrm{~ms}$ of two-way travel time error may result from this single interface interaction. While the full $10 \mathrm{~ms}$ is not likely to be the 
result of this one interaction, the wave must also pass through the sea floor twice adding another similar time-shift error.

The observed time shift may result in large relative RMS errors. Red and blue curves shown in Figure 12 represent the difference between 5 and $2.5 \mathrm{~m}$ solutions and 10 and 2.5 m solutions. Based on Richardson extrapolation (Kinkaid and Cheney (1996)) we estimate the relative RMS error to be about 7.5\%. However, comparing the red and blue curves to the $2.5 \mathrm{~m}$ solution, we see that the relative error at later times is much larger.

\section{DISCUSSION}

Existing works on simulation of wave propagation in discontinuous media show that the computational error associated with finite-difference methods consists of two components. One component grows with time and results in frequency-dependent grid dispersion. Application of higher-order methods suppresses the grid-dispersion error. The second component is due to misalignment between material interfaces and numerical grids. Theoretical analysis and numerical experiments presented in previous sections demonstrate that this first-order error manifests itself as a time-shift, results in large relative RMS error, and is insensitive to the order of the method. In the two-dimensional example, reduction in the overall error to $5 \%$ RMS appears to require 50 grid points per wavelength regardless of the order of the method.

Techniques that attempt to reduce the interface error require some kind of additional subgrid information about the coefficients or interface locations. Brown (1984) uses integral harmonic averaging to smooth the coefficients near the interfaces. Although this approach makes the interface error seconsd-order, its generalization to higher dimensions appears to be difficult. A multidimensional fourth-order scheme based on smoothed coefficients has been constructed and analyzed by Cohen and Joly (1996). They average coefficients using a smoothing parameter derived from energy estimates, and show that the approximation of reflection-transmission phenomena is an optimal, provided one chooses optimal smoothing parameter. In practice, a strategy for choice of the smoothing parameter relies on the information about points of discontinuity of the coefficients. Zhang and LeVeque (1997) apply the immersed interface approach to modify the difference scheme at grid points near the interfaces to achieve second order accuracy. In addition to the information about the 
interface locations, the immersed interface method requires knowledge about the jumps in the coefficients and their one-sided derivatives.

All the error-reducing methods mentioned above were developed in the context of regular-grid finite-difference schemes and cannot be applied directly to staggered schemes. Staggered grid, among other advantages, gives a compact difference scheme. Gustafsson and Mossberg (2004) proposed a fourth-order in time and space time-compact difference scheme based on Lax and Wendroff idea of using the differential equation to replace time derivatives by space derivatives. Analysis of this approach in the context of discontinuous media shows that it successfully controls grid dispersion, but does not eliminate the interface error (see Gustafsson and Wahlund (2004)).

\section{CONCLUSION}

In addition to a higher-order grid dispersion error, finite-difference simulation of wave propagation in discontinuous media is subject to the first-order error which is due to inaccurate interaction of the numerical wave with material interfaces. The grid dispersion error is well-understood and can be controlled by higher-order methods. In this paper, we concentrate on the quantitative evaluation of the interface misalignment error for a second-order in space and time staggered finite-difference scheme. Following Gustafsson and Wahlund (2004), we use plane wave analysis to study the numerical reflection and transmission coefficients. We show that the error is purely imaginary, which implies that the numerical and exact reflection and transmission coefficients have the same amplitude and differ only by a phase shift up to a second-order in $h \omega$. The phase shift is proportional to the time step and the distance between the interface and the main and staggered computational grids. Even if the interface is aligned with one of the grids, the misalignment with the second grid results in a non-zero error. Numerical examples support our theoretical analysis. In particular, the two-dimensional numerical experiment indicates that our one-dimensional analysis gives a good picture of what to expect in multi-dimensional computations and shows that the interfaces misalignment error cannot be controlled by higher-order methods alone. 


\section{REFERENCES}

Bourbie, T., O. Coussy, and B. Zinszner, 1987, Acoustics of porous media. Institut francais du petrole publications: Gulf Publishing Company. Translated from the French by Nissim Marshall.

Brown, D., 1984, A note on the numerical solution of the wave equation with piecewise smooth coefficients: Mathematics of Computation, 42, 369-391.

Cohen, G., 2002, Higher-order numerical methods for transient wave equations: SpringerVerlag.

Cohen, G. and P. Joly, 1996, Construction and analysis of fourth-order finite difference schemes for the acoustic wave equation in nonhomogeneous media: SIAM Journal on Numerical Analysis, 33, 1266-1302.

Dobrin, M. and C. Savit, 1988, Introduction to geophysical prospecting: McGraw-Hill Book Company, 4th edition.

Gustafsson, B. and E. Mossberg, 2004, Time compact high order difference methods for wave propagation: SIAM Journal on Scientific Computing, 26, 259-271.

Gustafsson, B. and P. Wahlund, 2004, Time compact difference methods for wave propagation in discontinuous media: SIAM Journal on Scientific Computing, 26, 272-293.

Kinkaid, D. and W. Cheney, 1996, Numerical analysis: Brooks-Cole, 2nd edition.

Virieux, J., 1984, SH-wave propagation in heterogeneous media: Velocity stress finitedifference method: Geophysics, 49, 1933-1957.

— 1986, P-SV wave propagation in heterogeneous media: Velocity stress finitedifference method: Geophysics, 51, 889-901.

Walden, A. and J. Hosken, 1986, The nature of the non-Gaussianity of primary reflection coefficients and its significance for deconvolution: Geophysical Prospecting, 34, 10381066.

Zhang, C. and R. J. LeVeque, 1997, The immersed interface method for acoustic wave equations with discontinuous coefficients: Wave Motion, 25, 237-263. 

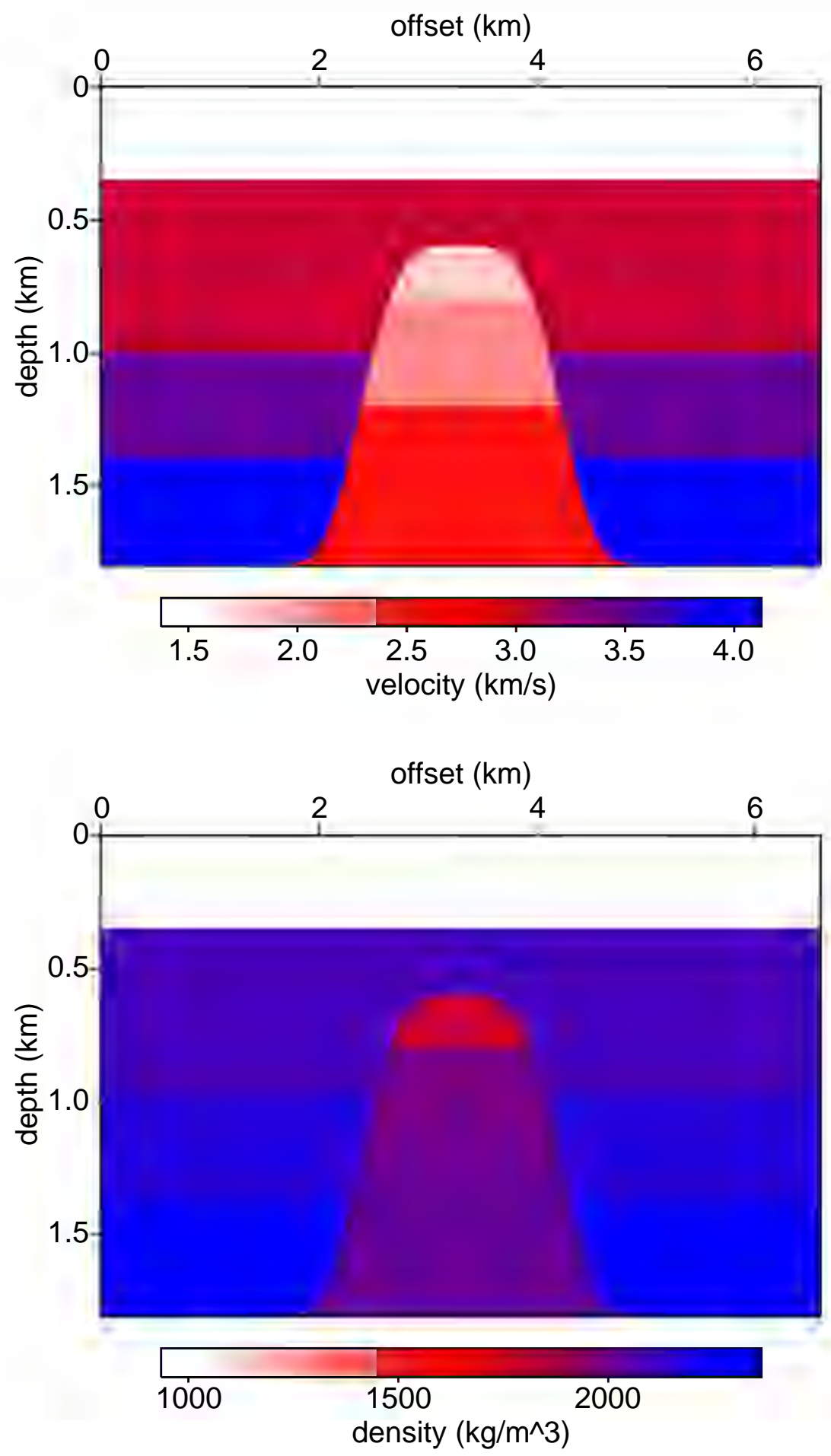

Figure 8: Velocity (top) and density (bottom) fields for Dome Model. 


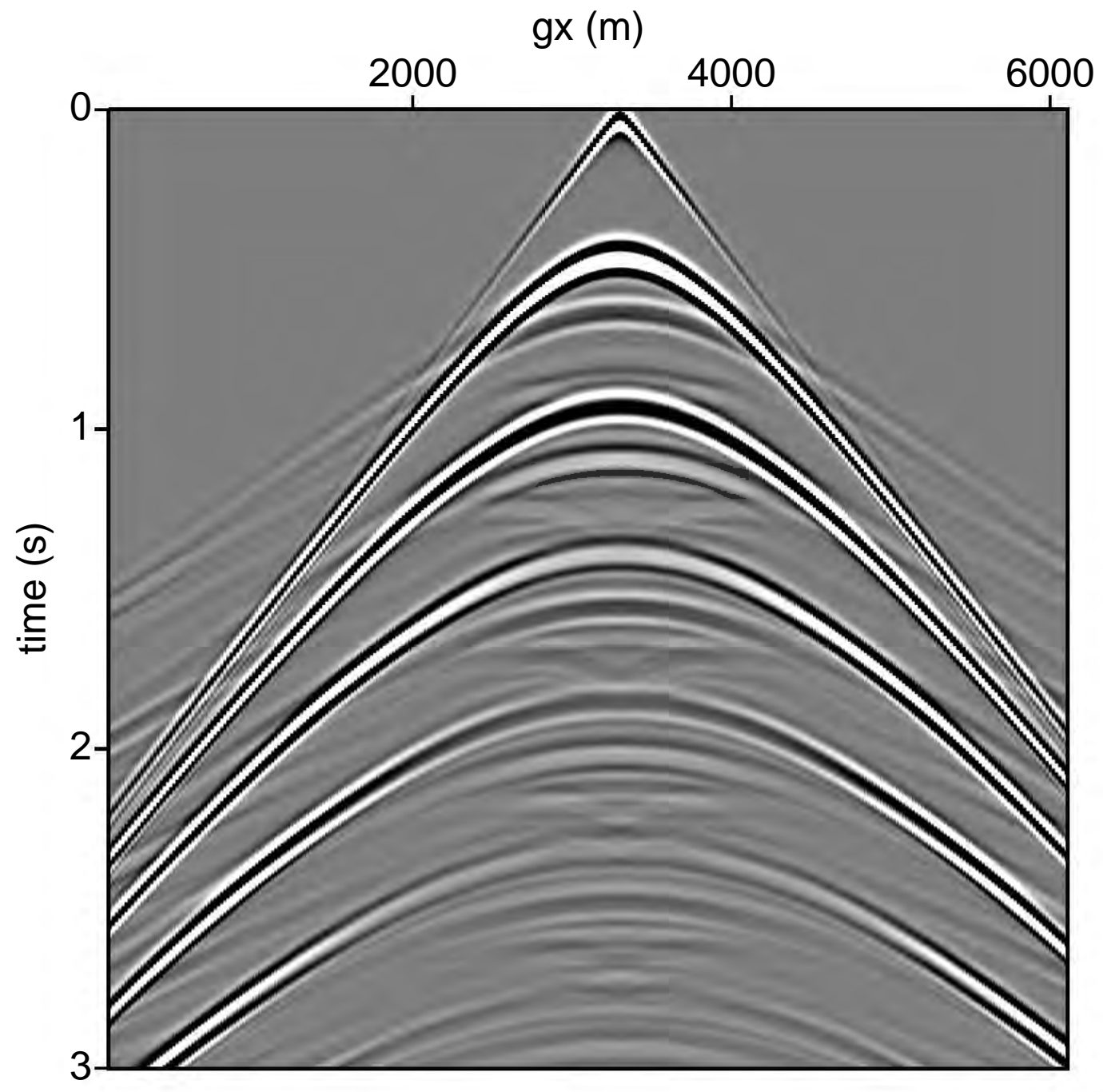

Figure 9: Simulated shot gather, square $10 \mathrm{~m}$ spatial grid, 2nd order in time 4th order in space staggered finite-difference scheme. 


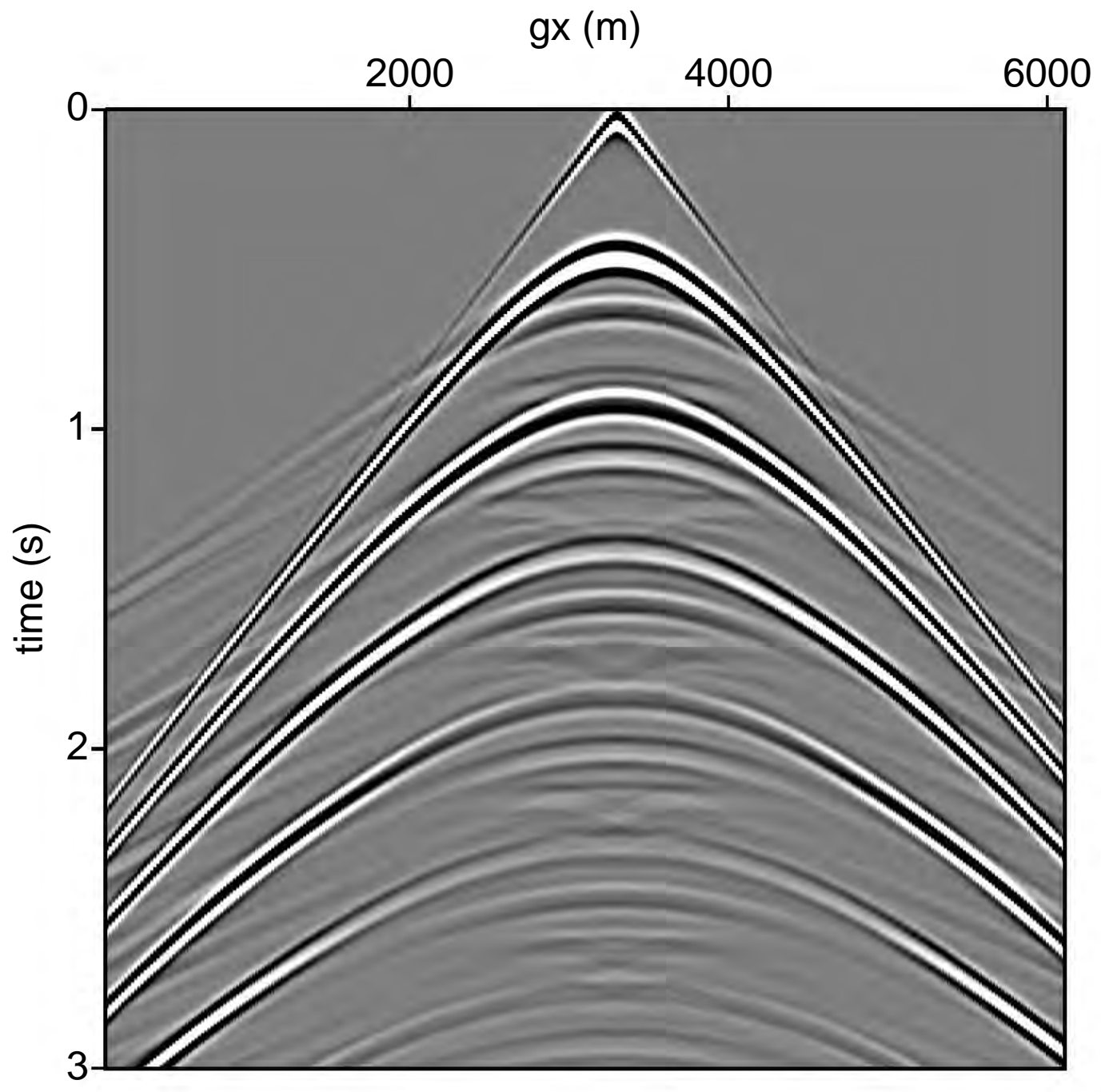

Figure 10: Simulated shot gather, square $10 \mathrm{~m}$ spatial grid, 2nd order in time 10th order in space staggered finite-difference scheme. 


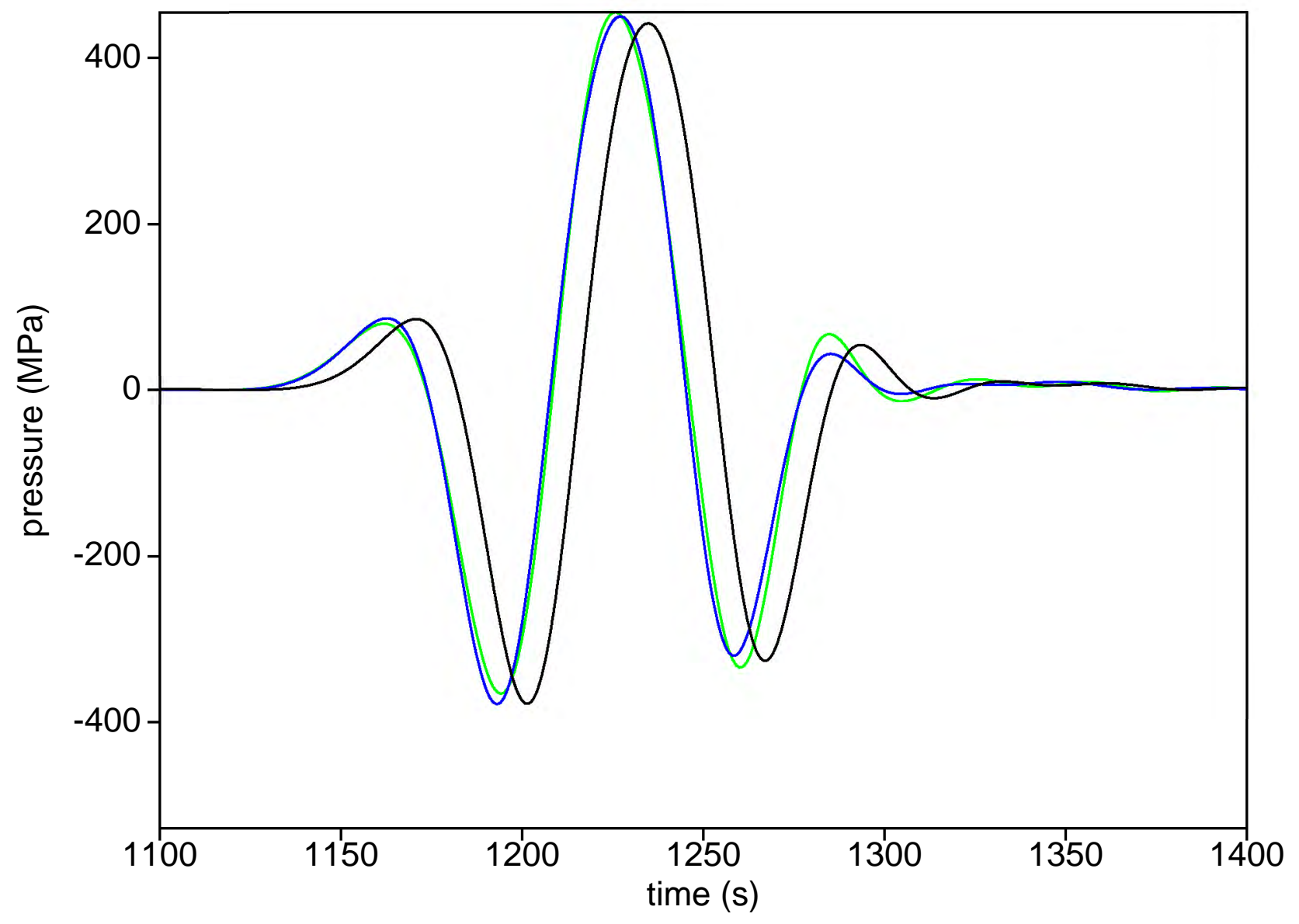

Figure 11: Trace detail at offset $1 \mathrm{~km}$. Green: $10 \mathrm{~m}$ grid, 4th order scheme. Blue: $10 \mathrm{~m}$ grid, 10th order scheme. Black: $2.5 \mathrm{~m}$ grid, 4th order scheme. 


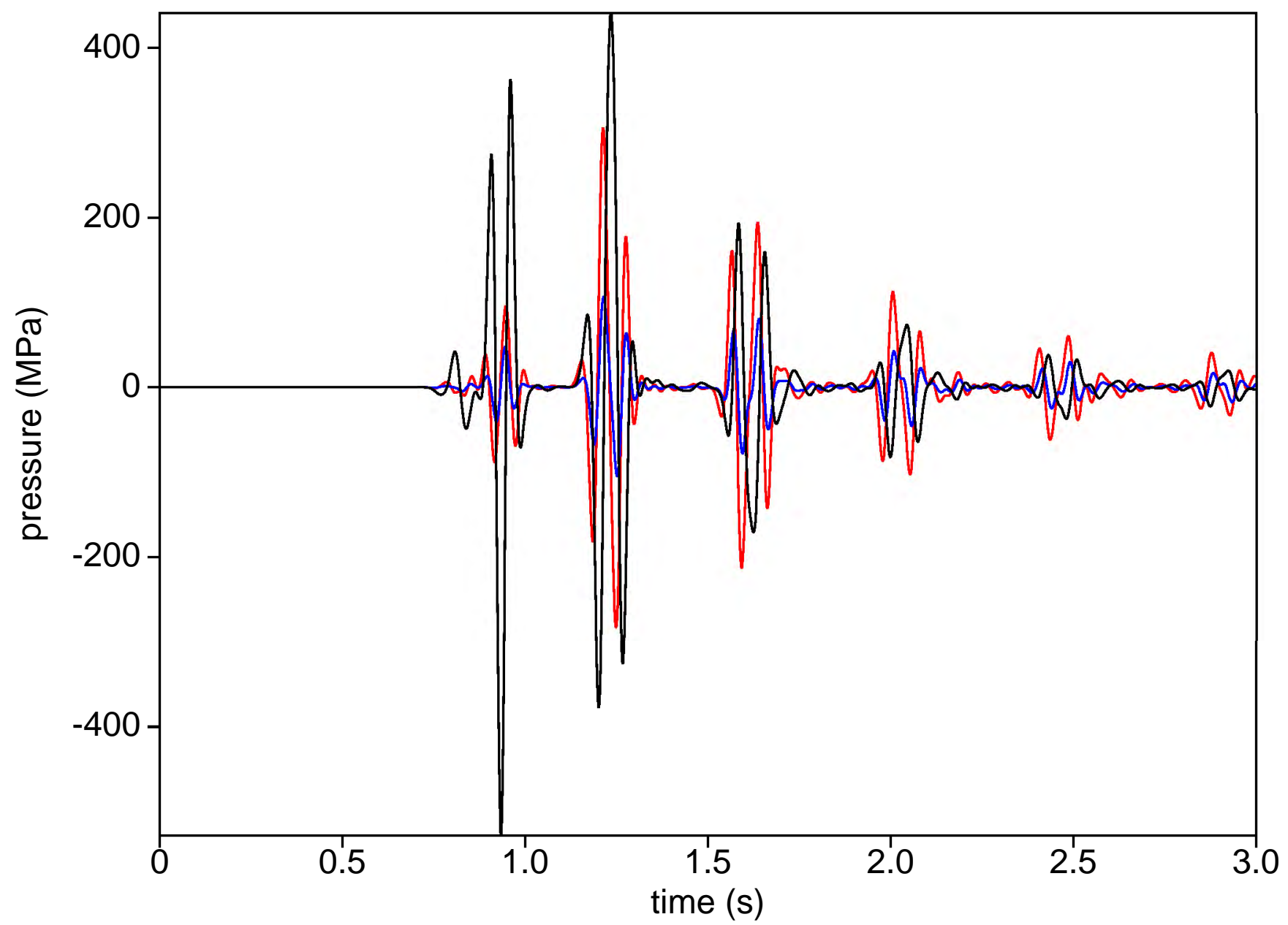

Figure 12: Comparison of traces for the 4th order scheme in two dimensions. Black: $2.5 \mathrm{~m}$ grid. Blue: difference between $5 \mathrm{~m}$ and $2.5 \mathrm{~m}$ solutions. Red: difference between $10 \mathrm{~m}$ and $2.5 \mathrm{~m}$ solutions. 